\title{
Electron energy-loss and inelastic X-ray scattering cross sections from time-dependent density-functional perturbation theory
}

\author{
Iurii Timrov and Nathalie Vast \\ Laboratoire des Solides Irradiés, École Polytechnique - CEA - DSM \\ - IRAMIS - CNRS UMR 7642, 91128 Palaiseau cedex, France
}

\author{
Ralph Gebauer \\ ICTP - The Abdus Salam International Centre for Theoretical Physics, Strada Costiera 11, 34014 Trieste, Italy
}

Stefano Baroni

Theory and Simulation of Materials (THEOS), École Polytechnique Fédérale de Lausanne, 1015 Lausanne, Switzerland and SISSA - Scuola Internazionale Superiore di Studi Avanzati, Via Bonomea 265, 34136 Trieste, Italy*

(Dated: June 24, 2021)

\begin{abstract}
The Liouville-Lanczos approach to linear-response time-dependent density-functional theory is generalized so as to encompass electron energy-loss and inelastic X-ray scattering spectroscopies in periodic solids. The computation of virtual orbitals and the manipulation of large matrices are avoided by adopting a representation of response orbitals borrowed from (time-independent) densityfunctional perturbation theory and a suitable Lanczos recursion scheme. The latter allows the bulk of the numerical work to be performed at any given transferred momentum only once, for a whole extended frequency range. The numerical complexity of the method is thus greatly reduced, making the computation of the loss function over a wide frequency range at any given transferred momentum only slightly more expensive than a single standard ground-state calculation, and opening the way to computations for systems of unprecedented size and complexity. Our method is validated on the paradigmatic examples of bulk silicon and aluminum, for which both experimental and theoretical results already exist in the literature.
\end{abstract}

PACS numbers: 31.15.ee, 71.45.Gm, 78.70.-g, 79.20.Uv

\section{INTRODUCTION}

Plasma oscillations in solids are possibly the simplest manifestation of collective effects in condensed matter, and their understanding in terms of plasmon modes one of the earliest triumphs of quantum many-body theory ${ }^{[1]}$ On the experimental side, collective chargedensity fluctuations can be probed through electron energy-loss (EEL) or inelastic X-Ray scattering (IXS) spectroscopies, two techniques that have been steadily producing a wealth of data since the early $60 \mathrm{~s}$ and $70 \mathrm{~s}$, respectively ${ }^{4 / 5}$ In the present day the engineering of novel materials down to the nanometer scale makes it possible to design devices where electromagnetic fields interact with collective oscillations of structures of subwavelength size. The strong dependence of plasmon dynamics on the size and shape of these nanostructures holds the promise of an extraordinary control over the optical response of the resulting devices, with applications to such diverse fields as photovoltaics $\left.{ }^{6}\right]$ proton beam acceleration,, 7 or biosensing,,$\frac{8}{}$ to name but a few. This is plasmonics, i.e photonics based on collective electronic excitations in strongly heterogeneous systems, where surface effects play a fundamental role. Plasma oscillations at surfaces have recently aroused a

\footnotetext{
*Permanent address
}

renewed attention by themselves, since it was shown that some metal surfaces unexpectedly exhibit acoustic plasmons. 14 These are collective charge excitations localized at the surface, whose frequency vanishes linearly with the wavevector, and are not damped by the bulk electron-hole continuum $\frac{15 \mid 16}{16}$ It is thought that these modes may offer the possibility of light confinement at designated locations on the surface, with possible applications in photonics and nano-optics 17

Most of the theoretical understanding of the optical response in nano-plasmonic systems relies on a classical approach: the nanostructure is usually described as an assembly of components, each characterized by an effective macroscopic dielectric function, and separated from the others by abrupt interfaces. The overall optical response is then computed by solving Maxwell's equation for the resulting heterogeneous system ${ }^{[18}$ When distances between the nanoscale components are themselves nanometric, however, quantum effects must be accounted for, and a fully quantum-mechanical description is called for.

Early quantum-mechanical approaches to the dynamics of charge-density fluctuations ${ }^{1-3}$ were based on the random-phase approximation as applied to the jellium model that, albeit exceedingly successful in simple metals and semiconductors, is not suitable for more complex materials, nor can it capture the fine, system-specific, features of even simple ones. The effects of crystal inhomogeneities on plasmon resonances in semiconductors (the so called local-field effects) were first addressed in the 
late $70 \mathrm{~s}, \frac{19}{19}$ using the empirical pseudopotential method, 20 along similar lines as previously followed for the optical spectra.21 In the present day the method of choice for describing charge dynamics in real materials (as opposed to simplified models, such as the jellium one) is timedependent (TD) density-functional theory (DFT) $\underline{22 \mid 23}$ Although some attempts to investigate EEL and IXS spectra using many-body perturbation theory have been made, 24 the vast majority of the studies existing to date relies on TDDFT, which in fact has been successfully used to study plasmons in a number of bulk ${ }^{28} 48$ and surface ${ }^{9}-14$ systems.

The conventional TDDFT approach to plasmon dynamics relies on the calculation of the charge-density susceptibility, $\chi$ (or, equivalently, inverse dielectric matrix, $\epsilon^{-1}$ ), starting from the independent-electron susceptibility, $\chi_{0}$, via a Dyson-like equation. $\stackrel{49}{ }$ Although successful in (relatively) simple systems that can be described by unit cells of moderate size, this methodology can hardly be applied to more complex systems, such as low-index or nano-structured surfaces, because of its intrinsic numerical limitations. In particular: $i$ ) the calculation of $\chi_{0}$ requires the knowledge of a large number of empty states, which is usually avoided in modern electronic-structure methods; ii) the solution of the Dyson-like equation requires the manipulation (multiplication and inversion) of (very) large matrices, and iii) all the above calculations have to be repeated independently for each value of the frequency to be sampled.

In this paper we introduce a new method, based on TD density-functional perturbation theory (DFPT), that allows to calculate EEL and IXS cross sections avoiding all the above drawbacks, and thus lending itself to numerical simulations in complex systems, potentially as large as several hundred independent atoms. Although the new methodology is general in principle, our implementation relies on the pseudopotential approximation, which limits its applicability to valence (or shallow-core) loss spectra. Inner-core loss spectra are currently addressed using different methods, as explained e.g. in Refs. 54 56. The salient features of our method are: $i$ ) the adoption of a representation from time-independent DFPT $\frac{57}{57}$ allows to avoid the calculation of Kohn-Sham (KS) virtual orbitals and of any large susceptibility matrices $\left(\chi\right.$ or $\left.\chi_{0}\right)$ altogether; and $\left.i i\right)$ thanks to the use of a Lanczos recursion scheme, the bulk of the calculations can be performed only once for all the frequencies simultaneously. The numerical complexity of the resulting algorithm is comparable, for the whole spectrum in a wide frequency range, to that of a single standard ground-state (or static response) calculation.

The paper is organized as follows. In Sec. II] we describe our basic theoretical and algorithmic frameworks, including the implementation of the newly proposed methodology for the response of a periodic system to a monochromatic perturbation, relevant to the calculation of EEL and IXS cross sections; in Sec. III we benchmark our technique on the prototypical examples of bulk silicon and aluminum, for which many experimental and well established theoretical results already exist; finally, our conclusions are presented in Sec. IV.

\section{THEORY AND ALGORITHMS}

Electron energy-loss spectroscopy probes the diffusion of a beam of fast electrons through a solid. According to Van Hove, 58 the corresponding double-differential cross section for inelastic scattering reads.

$$
\left(\frac{d^{2} \sigma}{d \Omega d \omega}\right)_{\mathrm{EEL}}=\left(\frac{4 \pi e^{2}}{Q^{2}}\right)^{2} \frac{m^{2}}{4 \pi^{2} \hbar^{4}} \frac{k_{f}}{k_{i}} S(\mathbf{Q}, \omega),
$$

where $-e$ and $m$ are the electron charge and mass, $\mathbf{k}_{i}, \mathbf{k}_{f}$, and $\mathbf{Q}=\mathbf{k}_{i}-\mathbf{k}_{f}$ are the incoming, outgoing, and transferred momenta, respectively, and $S(\mathbf{Q}, \omega)$ is the dynamic structure factor per unit volume.

While EEL spectroscopy is not suitable for samples enclosed in high-pressure cells, plasmon dynamics under pressure can be probed by IXS spectroscopy $[5960$ The double-differential cross-section reads in this case:

$$
\left(\frac{d^{2} \sigma}{d \Omega d \omega}\right)_{\mathrm{IXS}}=\left(\frac{e^{2}}{m c^{2}}\right)^{2}\left(\mathbf{e}_{i} \cdot \mathbf{e}_{f}\right)^{2} \frac{\omega_{f}}{\omega_{i}} S(\mathbf{Q}, \omega)
$$

where $\mathbf{e}_{i}$ and $\mathbf{e}_{f}$ are the incoming and scattered photon polarization directions, and $\omega_{i}$ and $\omega_{f}$ are the corresponding frequencies. According to the fluctuationdissipation theorem $S(\mathbf{Q}, \omega)$ is proportional to the imaginary part of the charge-density susceptibility, $\chi(\mathbf{Q}, \mathbf{Q} ; \omega)$ :

$$
S(\mathbf{Q}, \omega)=-\frac{\hbar}{\pi} \operatorname{Im} \chi(\mathbf{Q}, \mathbf{Q} ; \omega)
$$

In periodic solids the transferred momentum can be split into a component in the first Brillouin zone, $\mathbf{q}$, and a reciprocal-lattice vector, $\mathbf{G}$, as $\mathbf{Q}=\mathbf{q}+\mathbf{G}$, and $\chi$ is often expressed in terms of the inverse dielectric matrix, defined as! $62[63$

$$
\epsilon_{\mathbf{G G}^{\prime}}^{-1}(\mathbf{q}, \omega)=\delta_{\mathbf{G}, \mathbf{G}^{\prime}}+\frac{4 \pi e^{2}}{|\mathbf{q}+\mathbf{G}|^{2}} \chi\left(\mathbf{q}+\mathbf{G}, \mathbf{q}+\mathbf{G}^{\prime} ; \omega\right)
$$

where $\epsilon_{\mathbf{G G}^{\prime}}^{-1}(\mathbf{q}, \omega)=\epsilon^{-1}\left(\mathbf{Q}, \mathbf{Q}^{\prime} ; \omega\right)$. The function $-\operatorname{Im}\left[\epsilon^{-1}(\mathbf{Q}, \mathbf{Q} ; \omega)\right]$ is usually referred to as the loss function.

\section{A. Time-dependent density-functional perturbation theory}

In TDDFT electron dynamics is described by TD oneelectron equations for the occupied molecular orbitals. These TD KS equations read! 62

$$
i \frac{\partial \varphi_{v}(\mathbf{r}, t)}{\partial t}=\hat{H}_{\mathrm{KS}}(t) \varphi_{v}(\mathbf{r}, t)
$$


where $\varphi_{v}(\mathbf{r}, t)$ and $\hat{H}_{\mathrm{KS}}(t)$ are the TD KS orbitals and Hamiltonian (quantum mechanical operators are indicated with a caret), respectively, the index $v$ spans the $N_{v}$ occupied (valence) states, and atomic units $(e=m=$ $\hbar=1)$ are used henceforth. The KS Hamiltonian reads:

$$
\hat{H}_{\mathrm{KS}}(t)=-\frac{1}{2} \nabla^{2}+V_{\text {ext }}(\mathbf{r}, t)+V_{\mathrm{HXC}}(\mathbf{r}, t),
$$

where $V_{\text {ext }}(\mathbf{r}, t)$ and $V_{\mathrm{HXC}}(\mathbf{r}, t)$ are the external and Hartree-plus-exchange-correlation (HXC) potentials, respectively. Let us assume that the external potential can be split into a static term, plus a small TD perturbation:

$$
V_{\text {ext }}(\mathbf{r}, t)=V_{\text {ext }}^{\circ}(\mathbf{r})+\lambda(t) V_{\text {ext }}^{\prime}(\mathbf{r}),
$$

where $\lambda(t)$ is the TD strength of the perturbation. The total KS potential is perturbed accordingly: $V^{\prime}(\mathbf{r}, t)=$ $\lambda(t) V_{e x t}^{\prime}(\mathbf{r})+V_{\mathrm{HXC}}^{\prime}(\mathbf{r}, t), V_{\mathrm{HXC}}^{\prime}$ being the response $\mathrm{HXC}$ potential. The response of the KS orbitals is defined as

$$
\varphi_{v}(\mathbf{r}, t)=e^{-i \varepsilon_{v} t}\left(\varphi_{v}^{\circ}(\mathbf{r})+\varphi_{v}^{\prime}(\mathbf{r}, t)\right),
$$

$\varphi_{v}^{\circ}(\mathbf{r})$ and $\varepsilon_{v}$ being the unperturbed ground-state KS orbitals and energies, respectively. The charge-density susceptibility is the response of the electron charge density, which only depends on the projection of the response of the valence KS orbitals onto the empty-state (conduction) manifold. The Fourier transforms (indicated by tilde " " hereafter) of such projected response orbitals are obtained from standard first-order perturbation theory via the linear systems:

$$
\left(\hat{H}^{\circ}-\varepsilon_{v}-\omega\right) \tilde{\varphi}_{v}^{\prime}(\mathbf{r}, \omega)=-\hat{P}_{c} \tilde{V}^{\prime}(\mathbf{r}, \omega) \varphi_{v}^{\circ}(\mathbf{r}),
$$

where $\hat{P}_{c}$ is the projector over the unperturbed conduction-state manifold. Expressing the latter in terms of valence orbitals $\left(\hat{P}_{c}+\hat{P}_{v}=1\right)$ allows one to compute response KS orbitals without making any reference to unoccupied states, much in the same way as it is done in time-independent DFPT ${ }^{[57}$ The solution of Eq. (9) requires one to express the total response potential, $V^{\prime}(\mathbf{r}, \omega)$, in terms of its own solutions, through the response charge density, which is the diagonal of the response density matrix, $n^{\prime}(\mathbf{r}, t)=\rho^{\prime}(\mathbf{r}, \mathbf{r} ; t)$, whose Fourier transform is defined as:

$$
\begin{aligned}
\tilde{\rho}^{\prime}\left(\mathbf{r}, \mathbf{r}^{\prime} ; \omega\right)=2 \sum_{v=1}^{N_{v}}\left(\tilde{\varphi}_{v}^{\prime}(\mathbf{r}, \omega) \varphi_{v}^{\circ *}\left(\mathbf{r}^{\prime}\right)\right. & \\
& \left.+\varphi_{v}^{\circ}(\mathbf{r}) \tilde{\varphi}_{v}^{\prime *}\left(\mathbf{r}^{\prime},-\omega\right)\right),
\end{aligned}
$$

where the factor two accounts for spin degeneracy in a non-polarized system. Note that $\tilde{n}^{\prime}(\mathbf{r}, \omega)=\tilde{n}^{\prime *}(\mathbf{r},-\omega)$, as a consequence of the reality of $n^{\prime}(\mathbf{r}, t)$. The equation for the complex conjugate of $\tilde{\varphi}_{v}^{\prime}(\mathbf{r}, \omega)$ reads:

$$
\left(\hat{H}^{\circ}-\varepsilon_{v}+\omega\right) \tilde{\varphi}_{v}^{* *}(\mathbf{r},-\omega)=-\hat{P}_{c} \tilde{V}^{\prime}(\mathbf{r}, \omega) \varphi_{v}^{\circ *}(\mathbf{r}),
$$

where use has been made of the reality of the perturbing potential $\left(\tilde{V}^{\prime}(\omega)=\tilde{V}^{\prime *}(-\omega)\right)$. Equations (9) and (11) describe the resonant and anti-resonant contributions to charge-density response, respectively. Their left-hand sides just differ by the sign of the frequency, while, by using time-reversal symmetry of the unperturbed system $\left(\varphi_{v}^{\circ *}=\varphi_{v}^{\circ}\right)$ their right-hand side can be made look alike. The equations for the resonant and anti-resonant components of the charge-density response are coupled by the HXC potential, which is determined self-consistently by the density response itself, through the relation:

$$
\tilde{V}_{\mathrm{HXC}}^{\prime}(\mathbf{r}, \omega)=\int \kappa\left(\mathbf{r}, \mathbf{r}^{\prime}\right) \tilde{n}^{\prime}\left(\mathbf{r}^{\prime}, \omega\right) d \mathbf{r}^{\prime},
$$

where

$$
\kappa\left(\mathbf{r}, \mathbf{r}^{\prime}\right)=\frac{1}{\left|\mathbf{r}-\mathbf{r}^{\prime}\right|}+\frac{\delta V_{\mathrm{XC}}(\mathbf{r})}{\delta n\left(\mathbf{r}^{\prime}\right)}
$$

is the HXC kernel, which we assume to be independent of frequency, consistently with the adiabatic DFT approximation 64

The TD KS equations (5) can be equivalently expressed in terms of a quantum Liouville equation for the one-particle density matrix, $\hat{\rho}(t) ![51 / 53$

$$
i \frac{d \hat{\rho}(t)}{d t}=\left[\hat{H}_{\mathrm{KS}}(t), \hat{\rho}(t)\right] .
$$

Upon linearization and Fourier transformation, Eq. (14) takes the form:

$$
(\omega-\hat{\mathcal{L}}) \cdot \hat{\rho}^{\prime}(\omega)=\tilde{\lambda}(\omega)\left[\hat{V}_{e x t}^{\prime}, \hat{\rho}^{\circ}\right],
$$

where $\hat{\rho}^{\circ}$ is the unperturbed density matrix and $\hat{\mathcal{L}}$ is the Liouvillian super-operator, defined by the relation: $51[53$

$$
\hat{\mathcal{L}} \cdot \hat{\rho}^{\prime}=\left[\hat{H}^{\circ}, \hat{\rho}^{\prime}\right]+\left[\hat{V}_{\mathrm{HXC}}^{\prime}\left[\hat{\rho}^{\prime}\right], \hat{\rho}^{\circ}\right] .
$$

The response of an arbitrary one-electron Hermitian operator, $\hat{A}$, to an external perturbation, $\hat{V}_{e x t}$, is described by the generalized susceptibility:

$$
\begin{aligned}
\chi_{A V}(\omega) & \equiv \frac{1}{\tilde{\lambda}(\omega)} \operatorname{Tr}\left(\hat{A} \hat{\rho}^{\prime}(\omega)\right) \\
& =\left(\hat{A},(\omega-\hat{\mathcal{L}})^{-1} \cdot\left[\hat{V}_{\text {ext }}^{\prime}, \hat{\rho}^{0}\right]\right),
\end{aligned}
$$

where $(\cdot, \cdot)$ indicates a scalar product in an abstract operator manifold ${ }^{52}$ Equation (18) states that, within TDDFT, the most general susceptibility can be expressed as an off-diagonal element of the resolvent of the Liouvillian.

\section{B. The Liouville-Lanczos algorithm}

The calculation of susceptibilities from Eq. 18 requires the explicit representation of the response density matrix and of the Liouvillian super-operator acting on it. The minimum dimension of such a representation is $2 \times N_{v} \times N_{c}$, where $N_{c}=N-N_{v}$ is the 
number of virtual (conduction) orbitals and $N$ the dimension of one-electron basis set 65 The inversion of the Liouvillian appearing in Eq. 18 is a formidable task in typical large-scale plane-wave calculations, where the number of occupied states can be as large as several hundreds to a few thousands, and the number of virtual orbitals a hundred times as large. The recursion method by Haydock, Heine, and Kelly $\sqrt{66}$ offers an elegant solution to a similar problem, namely the calculation of a diagonal element of the resolvent of a Hermitian matrix, in terms of a continued fraction, whose coefficients are frequency-independent. The Lanczos biorthogonalization algorithm,, 515367 allows one to generalize this procedure to the calculation of off-diagonal elements of the resolvent of a non-Hermitian matrix. The resulting numerical workload for calculating the full spectrum in a whole wide frequency range is comparable to that of a single ground-state (or static response) calculation. Other flavours of the Lanczos-type algorithm can be found in Refs. 6869 .

\section{The Lanczos bi-orthogonalization algorithm}

We want to calculate matrix elements such as:

$$
g(\omega)=\left(u,(\omega-L)^{-1} v\right)
$$

where $L$ is a $P \times P$ non-Hermitian matrix, and $u$ and $v$ are generic $P$-dimensional arrays. To this end we define two sets of Lanczos vectors, $\left\{v_{j}\right\}$ and $\left\{u_{j}\right\}$, through the recursive relations! 67

$$
\begin{aligned}
& \beta_{j+1} v_{j+1}=L v_{j}-\alpha_{j} v_{j}-\gamma_{j} v_{j-1}, \\
& \gamma_{j+1} u_{j+1}=L^{\top} u_{j}-\alpha_{j} u_{j}-\beta_{j} u_{j-1},
\end{aligned}
$$

where one defines $u_{0}=v_{0}=0, u_{1}=v_{1}=v$, and the $\alpha_{j}$, $\beta_{j}$, and $\gamma_{j}$ Lanczos coefficients are determined by the $b i$ orthogonality conditions $\left(u_{j}, v_{j}\right)=1$, and $\left(u_{j-1}, v_{j}\right)=$ $\left(u_{j}, v_{j-1}\right)=0$. The set of vectors and coefficients generated through the recursion relations 20 21 is often referred to as a Lanczos chain. The details of this algorithm are reviewed e.g. in Ref. 67, and its specialization to TDDFT is presented in Refs. [51/53. For the purposes of the present paper, we limit ourselves to observe that the Lanczos vectors thus generated have the property that they provide a tridiagonal representation of the $L$ matrix. More specifically, if we define the $P \times M$ matrices ${ }^{M} U=\left\{u_{1}, u_{2}, \ldots, u_{M}\right\}$ and ${ }^{M} V=\left\{v_{1}, v_{2}, \ldots, v_{M}\right\}(M$ being the number of Lanczos iterations), one has:

$$
\left({ }^{M} U\right)^{\top} L{ }^{M} V={ }^{M_{T}}
$$

where ${ }^{M_{T}}$ is the tridiagonal matrix

$$
M_{T}=\left(\begin{array}{ccccc}
\alpha_{1} & \gamma_{2} & 0 & \ldots & 0 \\
\beta_{2} & \alpha_{2} & \gamma_{3} & 0 & \vdots \\
0 & \beta_{3} & \alpha_{3} & \ddots & 0 \\
\vdots & 0 & \ddots & \ddots & \gamma_{M} \\
0 & \ldots & 0 & \beta_{M} & \alpha_{M}
\end{array}\right)
$$

In this Lanczos representation, the matrix element of Eq. 19. can be expressed as:

$$
g(\omega) \simeq\left({ }^{M_{z}},\left(\omega^{M} I-{ }^{M_{T}}\right)^{-1} \cdot{ }^{M} e_{1}\right),
$$

where ${ }^{M} e_{1}=\{1,0, \ldots, 0\}$ and ${ }^{M_{z}}$ is the $M$-dimensional vector defined as:

$$
{ }^{M} z=\left({ }^{M} V\right)^{\top} u .
$$

The right-hand side of Eq. 24 can be conveniently computed by solving, for any given value of $\omega$, the equation:

$$
\left(\omega^{M} I-{ }^{M} T\right){ }^{M} x={ }^{M} e_{1},
$$

and calculating the scalar product:

$$
g(\omega)=\left({ }^{M_{z}},{ }^{M} x\right)
$$

The vector $M_{z}$, Eq. 25, can be computed on the fly during the Lanczos recursion, through the relation $z_{j}=$ $\left(u, v_{j}\right)$. In practice, the procedure outlined above is performed in two steps. In the first step, which is by far the most time consuming, one generates the tridiagonal matrix ${ }^{M_{T}}$, Eq. (23), and the vector ${ }^{M_{z}}$, Eq. 25). In the second step $g(\omega)$ is calculated from Eq. 27) upon the solution of Eq. 26), for different frequencies $\omega$. In practice, a small imaginary part $\eta$ is added to the frequency argument, $\omega \rightarrow \omega+i \eta$, so as to regularize the function $g(\omega) \stackrel{5153}{ }$ Setting $\eta$ to a non-zero value amounts to broadening each individual spectral line or, alternatively, to convoluting the function $g(\omega)$ with a Lorentzian. Because of the tridiagonal form and the small dimension of the matrix ${ }^{M_{T}}$ (a few hundreds to a few thousands), the second step is essentially gratis. Different responses to a same perturbation can be computed simultaneously from a same Lanczos recursion, by computing different $z$ vectors on the fly.

\section{The batch representation}

Equation (10) shows that the response density matrix is uniquely determined by the two sets of functions $\left\{\tilde{\varphi}_{v}^{\prime}(\mathbf{r}, \omega)\right\}$ and $\left\{\tilde{\varphi}_{v}^{*}(\mathbf{r},-\omega)\right\}$. It is convenient to consider a linear combination of these functions, defined as:

$$
\begin{aligned}
& q_{v}(\mathbf{r})=\frac{1}{2}\left(\tilde{\varphi}_{v}^{\prime}(\mathbf{r}, \omega)+\tilde{\varphi}_{v}^{\prime *}(\mathbf{r},-\omega)\right), \\
& p_{v}(\mathbf{r})=\frac{1}{2}\left(\tilde{\varphi}_{v}^{\prime}(\mathbf{r}, \omega)-\tilde{\varphi}_{v}^{\prime *}(\mathbf{r},-\omega)\right) .
\end{aligned}
$$


The two sets $\left\{q_{v}\right\}$ and $\left\{p_{v}\right\}$ are called respectively the $u p$ per and lower component of the standard batch representation (SBR) $\lcm{5153}$ of the response density matrix supervector: $\hat{\rho}^{\prime} \stackrel{\text { SBR }}{\longrightarrow}\left\{\left\{q_{v}\right\},\left\{p_{v}\right\}\right\} !^{52}$ The SBR of a Hermitian operator, $\hat{A}$, has vanishing lower component, $\hat{A} \stackrel{\mathrm{SBR}}{\longrightarrow}$ $\left\{\left\{\hat{P}_{c} \hat{A} \varphi_{v}^{\circ}(\mathbf{r})\right\}, 0\right\}$, while that of its commutator with the unperturbed density matrix [see Eq. [15]] has vanishing upper component, $\left[\hat{A}, \hat{\rho}^{\circ}\right] \stackrel{\mathrm{SBR}}{\longrightarrow}\left\{0,\left\{\hat{P}_{c} \hat{A} \varphi_{v}^{\circ}(\mathbf{r})\right\}\right\}$. The SBR of the Liouvillian super-operator has the block form:

$$
\hat{\mathcal{L}}=\left(\begin{array}{cc}
0 & \hat{\mathcal{D}} \\
\hat{\mathcal{D}}+\hat{\mathcal{K}} & 0
\end{array}\right)
$$

where the $\hat{\mathcal{D}}$ and $\hat{\mathcal{K}}$ super-operators are defined by their action on response batches,

$$
\begin{aligned}
\hat{\mathcal{D}}\left\{q_{v}(\mathbf{r})\right\} & =\left\{\left(\hat{H}^{\circ}-\varepsilon_{v}\right) q_{v}(\mathbf{r})\right\} \\
\hat{\mathcal{K}}\left\{q_{v}(\mathbf{r})\right\} & =\left\{4 \hat{P}_{c} \sum_{v^{\prime}} \int \kappa\left(\mathbf{r}, \mathbf{r}^{\prime}\right) \varphi_{v^{\prime}}^{\circ *}\left(\mathbf{r}^{\prime}\right) q_{v^{\prime}}\left(\mathbf{r}^{\prime}\right) d \mathbf{r}^{\prime} \varphi_{v}^{\circ}(\mathbf{r})\right\} \\
& =\left\{\hat{P}_{c} V_{\mathrm{HXC}}^{\prime}(\mathbf{r}) \varphi_{v}^{\circ}(\mathbf{r})\right\}
\end{aligned}
$$

$\kappa\left(\mathbf{r}, \mathbf{r}^{\prime}\right)$ is the HXC kernel of Eq. 13), and $V_{\mathrm{HXC}}^{\prime}$ is the HXC potential (see Eq. (12) generated by the response charge density distribution whose SBR is (see Eq. (10)):

$$
n^{\prime}(\mathbf{r})=4 \sum_{v} \varphi_{v}^{\circ *}(\mathbf{r}) q_{v}(\mathbf{r}) .
$$

According to the above equations, operating with the Liouvillian on a test super-vector essentially requires the calculation of the $\mathrm{HXC}$ potential response, its application to each valence $\mathrm{KS}$ orbital, as well as the operation of the unperturbed Hamiltonian onto twice the number of valence $\mathrm{KS}$ states.

The starting super-vector of the Lanczos recursion is the right-hand side of Eq. 15 whose SBR is:

$$
v_{1}=u_{1} \stackrel{\mathrm{SBR}}{\longrightarrow}\left(\begin{array}{c}
0 \\
\left\{\hat{P}_{c} \tilde{V}_{e x t}^{\prime}(\mathbf{r}) \varphi_{v}^{\circ}(\mathbf{r})\right\}
\end{array}\right) .
$$

Because of the special block structure of the Liouvillian, Eq. (30), the SBR of odd Lanczos iterates have vanishing upper components, whereas the even ones have vanishing lower components. As a consequence, the number of response wavefunctions onto which the unperturbed Hamiltonian must operate per Lanczos iteration is halved. Also, the diagonal elements of the resulting tridiagonal matrix (the $\alpha$ coefficients) are all vanishing.

\section{Lanczos-chain extrapolation}

It was previously noted that the components of the vector $M_{z}$, Eq. 25), decrease rather rapidly to zero, whereas the $\beta_{j}$ (and $\gamma_{j}$ ) coefficients oscillate around two distinct values for odd and even iterations, whose average is approximatively equal to one half of the kinetic-energy cutoff (in a plane-wave implementation), and whose difference is approximately twice as large as the excitation gap in insulating or semiconducting materials. ${ }^{5153}$ This finding can be used to speed up considerably the calculation by adopting a suitable extrapolation technique. In practice, the Lanczos recursion is stoped after $M_{0}$ iterations, such that the components of the $z$ array are small enough. The dimension $M$ of the linear system, Eq. 26, is then set to a very large (and to a large extent arbitrary) value. The $z$ components from $M_{0}+1$ to $M$ are set to zero, whereas the corresponding $\beta$ and $\gamma$ coefficients are set to the average of the values that have been actually computed. The accuracy of the calculated spectrum is then checked a posteriori with respect to the value of $M_{0}$. In many applications it turns out that $M_{0}$ may vary from a few hundreds up to a few thousands (depending on the plane-wave kinetic energy cutoff), and $M$ is a (to a large extent arbitrary) number reaching up to several thousands. As the solution of tridiagonal systems can be performed very efficiently via standard factorization techniques, the numerical overhead of this procedure is negligible. More on Lanczos extrapolation can be found in Refs. 51,53 .

\section{A Liouville-Lanczos approach to EEL and IXS spectroscopies in crystals}

In a periodic solid the unperturbed KS orbitals are $\varphi_{v}^{\circ}(\mathbf{r})=\varphi_{n, \mathbf{k}}^{\circ}(\mathbf{r})$, where $\{v\}=\{n, \mathbf{k}\}, n$ is a band index and $\mathbf{k}$ a point in the Brillouin zone. These KS orbitals can be cast into the Bloch form:

$$
\varphi_{n, \mathbf{k}}^{\circ}(\mathbf{r})=e^{i \mathbf{k} \cdot \mathbf{r}} u_{n, \mathbf{k}}^{\circ}(\mathbf{r}),
$$

where $u_{n, \mathbf{k}}^{\circ}(\mathbf{r})$ is the lattice-periodic function. Similarly, the total perturbing potential can be conveniently decomposed into Bloch components:

$$
\tilde{V}^{\prime}(\mathbf{r}, \omega)=\sum_{\mathbf{q}} \mathrm{e}^{i \mathbf{q} \cdot \mathbf{r}} \tilde{v}_{\mathbf{q}}^{\prime}(\mathbf{r}, \omega),
$$

where $\tilde{v}_{\mathbf{q}}^{\prime}(\mathbf{r})$ is also lattice-periodic, and the sum extends over the first Brillouin zone. A similar decomposition can be applied to the external and HXC response potentials. The response of each KS orbital can be correspondingly expressed as a linear combination of the responses to each Bloch component of the perturbing potential:

$$
\tilde{\varphi}_{n \mathbf{k}}^{\prime}(\mathbf{r}, \omega)=\sum_{\mathbf{q}} \mathrm{e}^{i(\mathbf{k}+\mathbf{q}) \cdot \mathbf{r}} \tilde{u}_{n, \mathbf{k}+\mathbf{q}}^{\prime}(\mathbf{r}, \omega),
$$

where $\tilde{u}_{n, \mathbf{k}+\mathbf{q}}^{\prime}(\mathbf{r}, \omega)$ is a lattice-periodic response orbital that satisfies the equation:

$$
\begin{aligned}
\left(\hat{H}_{\mathbf{k}+\mathbf{q}}^{\circ}-\varepsilon_{n, \mathbf{k}}-\omega\right) \tilde{u}_{n, \mathbf{k}+\mathbf{q}}^{\prime} & (\mathbf{r}, \omega)= \\
& -\hat{P}_{c}^{\mathbf{k}+\mathbf{q}} \tilde{v}_{\mathbf{q}}^{\prime}(\mathbf{r}, \omega) u_{n, \mathbf{k}}^{\circ}(\mathbf{r}) .
\end{aligned}
$$


In Eq. 38, as well as in the rest of this paper, quantummechanical operators bearing a wave-vector subscript (such as $\hat{H}_{\mathbf{k}+\mathbf{q}}^{\circ}$ ) or superscript (such as $\hat{P}_{c}^{\mathbf{k}+\mathbf{q}}$ ) are thought to operate on lattice-periodic functions, and are defined in terms of their coordinate representations as:

$$
\begin{aligned}
H^{\circ}\left(\mathbf{r}, \mathbf{r}^{\prime}\right) & =\sum_{\mathbf{k}} \mathrm{e}^{i \mathbf{k} \cdot\left(\mathbf{r}-\mathbf{r}^{\prime}\right)} H_{\mathbf{k}}^{\circ}\left(\mathbf{r}, \mathbf{r}^{\prime}\right) \\
P_{c}\left(\mathbf{r}, \mathbf{r}^{\prime}\right) & =\sum_{\mathbf{k}} \mathrm{e}^{i \mathbf{k} \cdot\left(\mathbf{r}-\mathbf{r}^{\prime}\right)} P_{c}^{\mathbf{k}}\left(\mathbf{r}, \mathbf{r}^{\prime}\right)
\end{aligned}
$$

The projector onto the conduction manifold in Eq. 38 can be expressed in terms of the periodic parts of the unperturbed Bloch functions as:

$$
P_{c}^{\mathbf{k}}\left(\mathbf{r}, \mathbf{r}^{\prime}\right)=\delta\left(\mathbf{r}-\mathbf{r}^{\prime}\right)-\sum_{n} u_{n, \mathbf{k}}^{\circ}(\mathbf{r}) u_{n, \mathbf{k}}^{\circ *}\left(\mathbf{r}^{\prime}\right),
$$

where the sum extends over all the occupied bands. A similar decomposition into Bloch components holds for the response density matrix, which reads in this case:

$$
\tilde{\rho}^{\prime}\left(\mathbf{r}, \mathbf{r}^{\prime} ; \omega\right)=\sum_{\mathbf{q}} \mathrm{e}^{i \mathbf{q} \cdot\left(\mathbf{r}-\mathbf{r}^{\prime}\right)} \tilde{\rho}_{\mathbf{q}}^{\prime}\left(\mathbf{r}, \mathbf{r}^{\prime}, \omega\right),
$$

where

$$
\begin{aligned}
\tilde{\rho}_{\mathbf{q}}^{\prime}\left(\mathbf{r}, \mathbf{r}^{\prime} ; \omega\right)=2 \sum_{n, \mathbf{k}}\left(\tilde{u}_{n, \mathbf{k}+\mathbf{q}}^{\prime}(\mathbf{r}, \omega) u_{n, \mathbf{k}}^{\circ *}\left(\mathbf{r}^{\prime}\right)+\right. \\
\left.u_{n, \mathbf{k}}^{\circ *}(\mathbf{r}) \tilde{u}_{n,-\mathbf{k}-\mathbf{q}}^{\prime *}\left(\mathbf{r}^{\prime},-\omega\right)\right) .
\end{aligned}
$$

The anti-resonant contribution to the density-matrix response in Eq. 43 satisfies the equation:

$$
\begin{aligned}
\left(\hat{H}_{\mathbf{k}+\mathbf{q}}^{\circ}-\varepsilon_{n, \mathbf{k}}+\omega\right) & \tilde{u}_{n,-\mathbf{k}-\mathbf{q}}^{\prime *}(\mathbf{r},-\omega)= \\
& -\hat{P}_{c}^{\mathbf{k}+\mathbf{q}} \tilde{v}_{\mathbf{q}}^{\prime}(\mathbf{r}, \omega) u_{n, \mathbf{k}}^{\circ}(\mathbf{r}),
\end{aligned}
$$

which can be obtained from Eq. (38) by complex conjugation and simple manipulations deriving from timereversal invariance of the unperturbed system $\left(u_{n, \mathbf{k}}^{\circ}=\right.$ $\left.u_{n,-\mathbf{k}}^{\circ *}\right)$ and the reality of the perturbing potential $\left(\tilde{v}_{\mathbf{q}}^{\prime}(\mathbf{r}, \omega)=\tilde{v}_{-\mathbf{q}}^{\prime *}(\mathbf{r},-\omega)\right)$.

\section{Batch representation for periodic solids}

In analogy with Eq. 10, Eq. (43) shows that the response density matrix of a periodic solid to a perturbation of wave-vector $\mathbf{q}$ is uniquely determined by the two sets of response orbitals $\left\{\tilde{u}_{n, \mathbf{k}+\mathbf{q}}^{\prime}(\mathbf{r}, \omega)\right\}$ and $\left\{\tilde{u}_{n,-\mathbf{k}-\mathbf{q}}^{\prime *}(\mathbf{r},-\omega)\right\}$. Note that $n$ and $\mathbf{k}$ are running indices, whereas $\mathbf{q}$ is fixed. The SBR can in this case be defined as:

$$
\begin{aligned}
& q_{n, \mathbf{k}+\mathbf{q}}(\mathbf{r})=\frac{1}{2}\left(\tilde{u}_{n, \mathbf{k}+\mathbf{q}}^{\prime}(\mathbf{r}, \omega)+\tilde{u}_{n,-\mathbf{k}-\mathbf{q}}^{\prime *}(\mathbf{r},-\omega)\right) \\
& p_{n, \mathbf{k}+\mathbf{q}}(\mathbf{r})=\frac{1}{2}\left(\tilde{u}_{n, \mathbf{k}+\mathbf{q}}^{\prime}(\mathbf{r}, \omega)-\tilde{u}_{n,-\mathbf{k}-\mathbf{q}}^{\prime *}(\mathbf{r},-\omega)\right)
\end{aligned}
$$

The two sets of response orbitals, $q_{\mathbf{q}}=\left\{q_{n, \mathbf{k}+\mathbf{q}}\right\}$ and $p_{\mathbf{q}}=\left\{p_{n, \mathbf{k}+\mathbf{q}}\right\}$ satisfy the coupled set of equations:

$$
\left(\begin{array}{cc}
\omega & -\hat{\mathcal{D}}_{\mathbf{q}} \\
-\hat{\mathcal{D}}_{\mathbf{q}}-\hat{\mathcal{K}}_{\mathbf{q}} & \omega
\end{array}\right)\left(\begin{array}{c}
q_{\mathbf{q}} \\
p_{\mathbf{q}}
\end{array}\right)=\left(\begin{array}{c}
0 \\
y_{\mathbf{q}}
\end{array}\right),
$$

where $y_{\mathbf{q}}=\left\{\hat{P}_{c}^{\mathbf{k}+\mathbf{q}} \tilde{v}_{e x t, \mathbf{q}}^{\prime}(\mathbf{r}) u_{n, \mathbf{k}}^{\circ}(\mathbf{r})\right\}$, and $\hat{\mathcal{D}}_{\mathbf{q}}$ and $\hat{\mathcal{K}}_{\mathbf{q}}$ are the super-operators defined by the relations:

$$
\begin{aligned}
& \hat{\mathcal{D}}_{\mathbf{q}} q_{\mathbf{q}}=\left\{\left(\hat{H}_{\mathbf{k}+\mathbf{q}}^{\circ}-\varepsilon_{n, \mathbf{k}}\right) q_{n, \mathbf{k}+\mathbf{q}}(\mathbf{r})\right\} \\
& \hat{\mathcal{K}}_{\mathbf{q}} q_{\mathbf{q}}=\left\{\hat{P}_{c}^{\mathbf{k}+\mathbf{q}} \tilde{v}_{\mathrm{HXC}, \mathbf{q}}^{\prime}(\mathbf{r}) u_{n, \mathbf{k}}^{\circ}(\mathbf{r})\right\},
\end{aligned}
$$

and

$$
\tilde{v}_{\mathrm{HXC}, \mathbf{q}}^{\prime}(\mathbf{r})=\int \kappa\left(\mathbf{r}, \mathbf{r}^{\prime}\right) n_{\mathbf{q}}^{\prime}\left(\mathbf{r}^{\prime}\right) d \mathbf{r}^{\prime},
$$

is the $\mathrm{HXC}$ potential generated by the response charge density:

$$
n_{\mathbf{q}}^{\prime}(\mathbf{r})=4 \sum_{n, \mathbf{k}} u_{n, \mathbf{k}}^{\circ *}(\mathbf{r}) q_{n, \mathbf{k}+\mathbf{q}}(\mathbf{r}) .
$$

Equations (48), 49, and (51) are closely parallel to Eqs. (31), (32), and (33) of Sec. II B 2

In practice, the sum over $\mathbf{k}$ points is limited to the portion of the Brillouin zone that is irreducible with respect to the small group of $\mathbf{q}$ and the resulting function symmetrized accordingly, in close analogy with timeindependent DFPT for lattice-dynamical calculations $\underline{57}$ More about the exploitation of crystal symmetry in the calculation of dynamical charge-density susceptibilities can be found in Ref. 70 .

The $\chi(\mathbf{Q}, \mathbf{Q} ; \omega)$ component of the charge-density susceptibility is obtained from Eq. (18) as the response of the $\mathbf{Q}=\mathbf{q}+\mathbf{G}$ Fourier component of the chargedensity operator, whose coordinate representation reads $\hat{n}(\mathbf{q}+\mathbf{G}) \rightarrow \mathrm{e}^{i(\mathbf{q}+\mathbf{G}) \cdot \mathbf{r}}$, to a monochromatic perturbation, $V_{\text {ext }}^{\prime}(\mathbf{r})=\mathrm{e}^{i(\mathbf{q}+\mathbf{G}) \cdot \mathbf{r}}$. The SBR of the periodic part of $\hat{n}(\mathbf{q}+\mathbf{G})$ is $\left\{\left\{\hat{P}_{c}^{\mathbf{k}+\mathbf{q}} \mathrm{e}^{i \mathbf{G} \cdot \mathbf{r}} u_{n, \mathbf{k}}^{\circ}\right\}, 0\right\}$. The final expression for the susceptibility is:

$$
\chi(\mathbf{Q}, \mathbf{Q} ; \omega)=\left(\left\{\left\{\hat{P}_{c}^{\mathbf{k}+\mathbf{q}} \mathrm{e}^{i \mathbf{G} \cdot \mathbf{r}} u_{n, \mathbf{k}}^{0}\right\}, 0\right\},\left\{q_{\mathbf{q}}, p_{\mathbf{q}}\right\}\right),
$$

where $\left\{q_{\mathbf{q}}, p_{\mathbf{q}}\right\}$ is the solution of Eq. 47), obtained when the periodic part of the external perturbing potential is $\tilde{v}_{\text {ext }, \mathbf{q}}^{\prime}(\mathbf{r})=\mathrm{e}^{i \mathbf{G} \cdot \mathbf{r}}$.

In practice the susceptibility in Eq. 52 is computed following the procedure outlined in Sec. II B 1] (see Eq. (24)):

$$
\chi(\mathbf{Q}, \mathbf{Q} ; \omega) \simeq\left({ }^{M_{z}},\left(\omega^{M} I-{ }^{M} T_{\mathbf{q}}\right)^{-1} \cdot{ }^{M} e_{1}\right)
$$

where ${ }^{M} T_{\mathbf{q}}$ is a tridiagonal matrix of dimension $M$ of the form 23, and $M_{z_{\mathbf{q}}}=\left(z_{1, \mathbf{q}}, z_{2, \mathbf{q}}, \ldots, z_{M, \mathbf{q}}\right)$ is an $M$ dimensional array whose coefficients $z_{j, \mathbf{q}}$ are defined as:

$$
z_{j, \mathbf{q}}=\left(\left\{\left\{\hat{P}_{c}^{\mathbf{k}+\mathbf{q}} e^{i \mathbf{G} \cdot \mathbf{r}} u_{n, \mathbf{k}}^{\circ}(\mathbf{r})\right\}, 0\right\}, v_{j}\right)
$$




\section{Metals}

The Liouville-Lanczos approach for EEL and IXS spectroscopies can be extended to metals by a suitable generalization of the smearing technique introduced by de Gironcoli in the static case for latticedynamical calculations ${ }^{[57 / 71}$ In the smearing approach, each KS energy level is broadened by a smearing function $(1 / \sigma) \tilde{\delta}(\varepsilon / \sigma)$, which is an approximation to the Dirac $\delta$-function in the limit of vanishing smearing width $\sigma$. The monochromatic $\mathbf{q}$ component of the charge-density response Eq. (51) can then be cast into the form:

$$
\begin{aligned}
n_{\mathbf{q}}^{\prime}(\mathbf{r})=2 \sum_{n, \mathbf{k}} u_{n, \mathbf{k}}^{\circ *}(\mathbf{r})\left(\bar{u}_{n, \mathbf{k}+\mathbf{q}}^{\prime}(\mathbf{r}, \omega)\right. & \\
+ & \left.\bar{u}_{n,-\mathbf{k}-\mathbf{q}}^{* *}(\mathbf{r},-\omega)\right),
\end{aligned}
$$

where the functions $\bar{u}_{n, \mathbf{k}+\mathbf{q}}^{\prime}(\mathbf{r}, \omega)$ and $\bar{u}_{n,-\mathbf{k}-\mathbf{q}}^{\prime *}(\mathbf{r},-\omega)$ satisfy the equations:

$$
\begin{aligned}
&\left(\hat{H}_{\mathbf{k}+\mathbf{q}}^{\circ}-\varepsilon_{n, \mathbf{k}}-\omega\right) \bar{u}_{n, \mathbf{k}+\mathbf{q}}^{\prime}(\mathbf{r}, \omega)= \\
&-\left(\tilde{\theta}_{F ; n, \mathbf{k}}-\hat{P}_{n, \mathbf{k}}^{\mathbf{k}+\mathbf{q}}\right) \tilde{v}_{\mathbf{q}}^{\prime}(\mathbf{r}, \omega) u_{n, \mathbf{k}}^{\circ}(\mathbf{r}), \\
&\left(\hat{H}_{\mathbf{k}+\mathbf{q}}^{\circ}-\varepsilon_{n, \mathbf{k}}+\omega\right) \bar{u}_{n,-\mathbf{k}-\mathbf{q}}^{\prime *}(\mathbf{r},-\omega)= \\
&-\left(\tilde{\theta}_{F ; n, \mathbf{k}}-\hat{P}_{n, \mathbf{k}}^{\mathbf{k}+\mathbf{q}}\right) \tilde{v}_{\mathbf{q}}^{\prime}(\mathbf{r}, \omega) u_{n, \mathbf{k}}^{\circ}(\mathbf{r})
\end{aligned}
$$

(cf. with Eqs. (38) and (44), where

$$
\begin{aligned}
\hat{P}_{n, \mathbf{k}}^{\mathbf{k}+\mathbf{q}} & =\sum_{m}^{o c c} \beta_{n, \mathbf{k} ; m, \mathbf{k}+\mathbf{q}}\left|u_{m, \mathbf{k}+\mathbf{q}}^{\circ}\right\rangle\left\langle u_{m, \mathbf{k}+\mathbf{q}}^{\circ}\right|, \\
\beta_{n, \mathbf{k} ; m, \mathbf{k}+\mathbf{q}}= & \tilde{\theta}_{F ; n, \mathbf{k}} \tilde{\theta}_{n, \mathbf{k} ; m, \mathbf{k}+\mathbf{q}} \\
+ & \tilde{\theta}_{F ; m, \mathbf{k}+\mathbf{q}} \tilde{\theta}_{m, \mathbf{k}+\mathbf{q} ; n, \mathbf{k}},
\end{aligned}
$$

$\tilde{\theta}_{F ; n, \mathbf{k}} \equiv \tilde{\theta}\left[\left(\varepsilon_{F}-\varepsilon_{n, \mathbf{k}}\right) / \sigma\right]$ and $\tilde{\theta}_{m, \mathbf{k}+\mathbf{q} ; n, \mathbf{k}} \equiv \tilde{\theta}\left[\left(\varepsilon_{m, \mathbf{k}+\mathbf{q}}-\right.\right.$ $\left.\left.\varepsilon_{n, \mathbf{k}}\right) / \sigma\right]$ being smooth approximations to the stepfunction, and $\varepsilon_{F}$ is the Fermi energy. It can be easily verified that the coefficients $\beta_{n, \mathbf{k} ; m, \mathbf{k}+\mathbf{q}}$ vanish when any of its indices refers to an unoccupied state. Therefore, the operator $\hat{P}_{n, \mathbf{k}}^{\mathbf{k}+\mathbf{q}}$ involves only a small number of partially occupied bands, and the first-order variation of the wavefunctions and of the charge density can be computed avoiding any explicit reference to unoccupied states, much in the same way as for insulating materials. More details about the Liouville-Lanczos approach for metals can be found in Ref. 70 .

\section{APPLICATION TO BULK SI AND AL}

The technique described above has been implemented in the QUANTUM ESPRESSO suite of computer codes,$\frac{722}{12}$ and is scheduled to be distributed in one of its future releases. We now proceed to validate it by calculating the loss function in bulk silicon and aluminum, for which several TDDFT studies exist, and whose spectra are known to be accurately described within the adiabatic local density (LDA) and generalized gradient (GGA) approximations (see, e.g., Refs. 4246 for $\mathrm{Si}$, and 2647 for $\mathrm{Al})$.

All the calculations have been performed within the LDA approximation, using the Perdew-Zunger parameterization of the electron-gas data, ${ }^{73}$ norm-conserving pseudopotentials from the QUANTUM ESPRESSO database ${ }^{74}$ and plane-wave basis sets up to a kineticenergy cutoff of $16 \mathrm{Ry}$. The first Brillouin zone has been sampled with a Monkhorst-Pack (MP) $\mathbf{k}$ point mesh, supplemented, in the case of Al, by the MethfesselPaxton smearing technique ${ }^{75}$ with a broadening parameter $\sigma=0.02 \mathrm{Ry}$. The frequency argument of the susceptibility has been assumed to have a small imaginary part, $\eta$, thus resulting in a Lorentzian smearing of the spectra (see Sec. IIB 1). For both $\mathrm{Si}$ and $\mathrm{Al}$ we have used the experimental lattice parameters (10.26 a.u ${ }^{76}$ and 7.60 a.u., ${ }^{77}$ respectively), which is very close to the theoretical one and resulting in no appreciable difference in the computed spectra.

\section{A. Bulk silicon}

Figure 1(a) shows the convergence of the loss spectrum of $\mathrm{Si}$, as calculated for a transferred momentum $Q=0.53$ a.u. along the [100] direction, as a function of the number of Lanczos iterations. After 400 iterations the spectrum displays spurious wiggles, which disappear by increasing the number of iterations up to 1500 . Also displayed are results obtained by the extrapolation procedure outlined at the end of Sec. IIB2, performed with $M_{0}=400$ Lanczos iterations and extrapolating the results up to a linear system of dimension $M=5000$. We see that the numerical workload can be considerably reduced without any appreciable loss of accuracy. In Fig. 1(b) we show the convergence of the loss function with respect to the $\mathbf{k}$ point sampling of the Brillouin zone. The $4 \times 4 \times 4$ MP $\mathbf{k}$ point mesh is not dense enough to obtain a well-converged spectrum, due to the presence of spurious wiggles, which disappear by increasing the size of the MP mesh up to $10 \times 10 \times 10$.

In Fig. 2 we compare our present results with those obtained from the conventional approach based on the Dyson-like equation for the susceptibility $46 / 49$ and with experiment. ${ }^{46}$ The agreement is excellent in both cases. All the salient features observed in the experiments at small transferred momentum (panel (a)) are correctly predicted: the main plasmon peak around $20 \mathrm{eV}$, a shoulder around $15 \mathrm{eV}$, and a weak peak around $6.5 \mathrm{eV}$. We attribute the slight differences between the two theoretical spectra to the slightly different technical details used in the two works. In particular, the authors of Ref. 46 mimicked electron- and hole-lifetime effects with an energy-dependent broadening, in contrast to the constant Lorentzian broadening, $\eta=0.035 \mathrm{Ry}$, used in our 


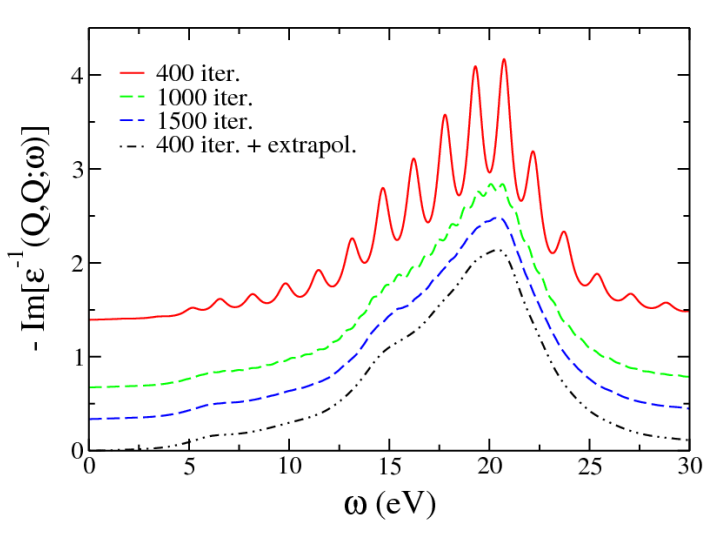

(a)

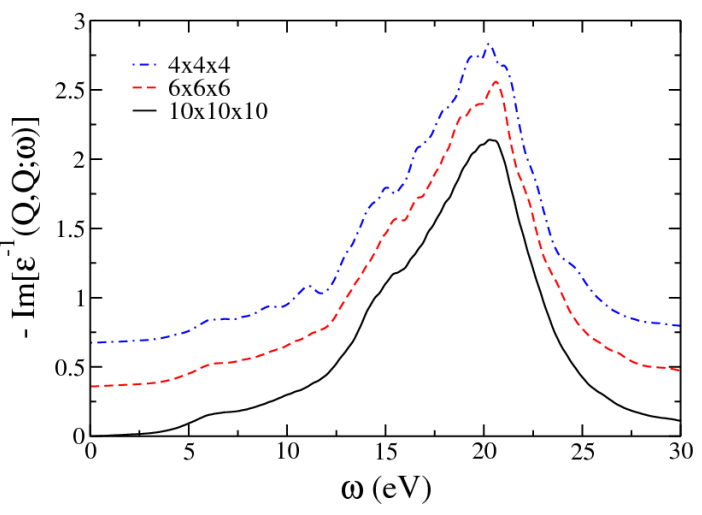

(b)

FIG. 1: Bulk Si: Loss function at $Q=0.53$ a.u. along [100]. (a) Convergence with respect to the number of Lanczos iterations, and effect of the extrapolation technique, using a $10 \times 10 \times 10$ Monkhorst-Pack k point mesh. (b) Convergence with respect to the size of the $\mathbf{k}$ point mesh, for 1500 Lanczos iterations. Both figures have been obtained with a Lorentzian broadening $\eta=0.035 \mathrm{Ry}$. Curves have been shifted vertically for clarity.

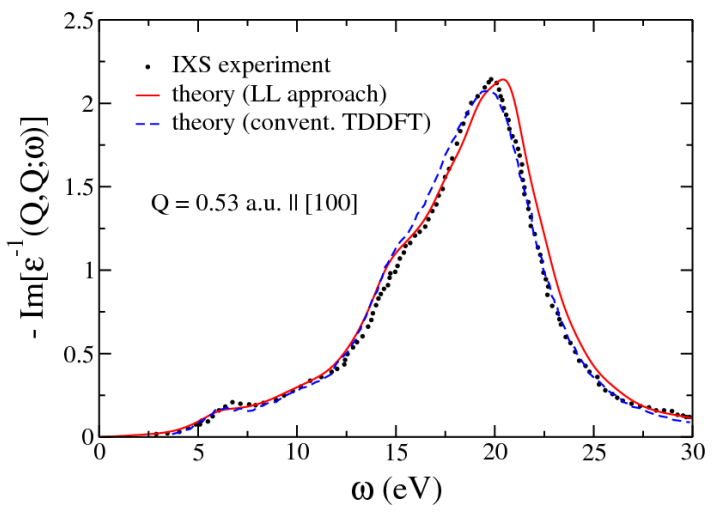

(a)

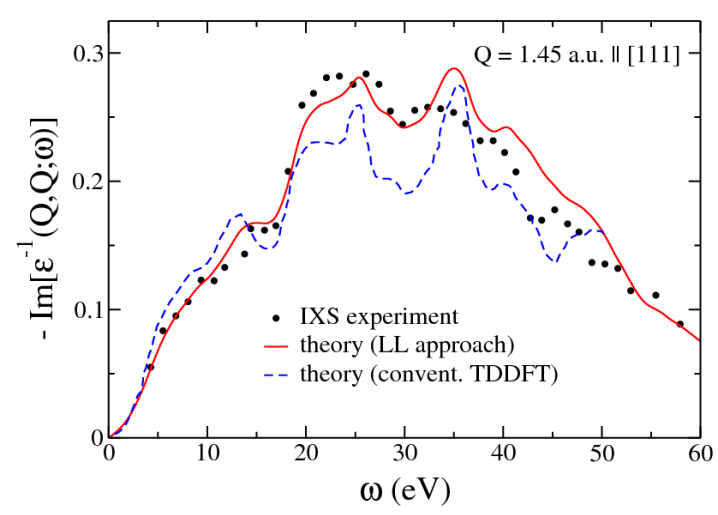

(b)

FIG. 2: Bulk Si: Comparison of the loss function calculated for two different values of the transferred momentum, Q, using the Liouville-Lanczos (LL) approach, with experiment ${ }^{46}$ and with previous calculations. $\frac{46}{\text { (a) }} Q=0.53$ a.u. along [100], (b) $Q=1.45$ a.u. along [111]. LL data have been obtained using 400 Lanczos iterations plus extrapolations. A $10 \times 10 \times 10$ $(6 \times 6 \times 6)$ MP k point mesh, and a Lorentzian broadening $\eta$ of $0.035(0.080)$ Ry have been used for the two cases, respectively.

calculations. At larger momentum transfer (panel (b)) the interaction of the plasmon with the electron-hole continuum broadens the spectrum. 78 The agreement with experiment, ${ }^{79}$ remarkable also in this case, is enhanced by increasing the Lorentzian broadening up to $\eta=0.080 \mathrm{Ry}$, which allowed us to reduce the size of the MP mesh down to $6 \times 6 \times 6$ without any appreciable loss of accuracy.

\section{B. Bulk aluminum}

Figure 3(a) shows the convergence of the loss function of Al, calculated at a transferred momentum $Q=$ 0.513 a.u. along the [100] direction, as a function of the number of Lanczos iterations. Although the qualitative behavior is similar to that observed in Si (wiggles showing up for a small number of iterations disappear by increasing this number), the convergence appears to be faster in the present case. As for the large-iterate behavior of the Lanczos coefficient, we observe that, in contrast to $\mathrm{Si}$, in $\mathrm{Al}$ the odd and even coefficients oscillate around a same value, which is also in this case of the order of one half the plane-wave kinetic-energy cutoff. This is due to the vanishing of the gap, as discussed in Ref. 51 53. Figure 3(b) shows the convergence with respect to the size of the $\mathbf{k}$ point mesh: very satisfactory convergence is achieved with $10 \times 10 \times 10 \mathrm{MP}$ mesh and a broadening parameter $\eta=0.056 \mathrm{Ry}$.

In Fig. 4 we compare the loss function of $\mathrm{Al}$ as calculated by the present method for two different values of the transferred momentum along the [100] direction, with IXS experiments and with previous theoretical 


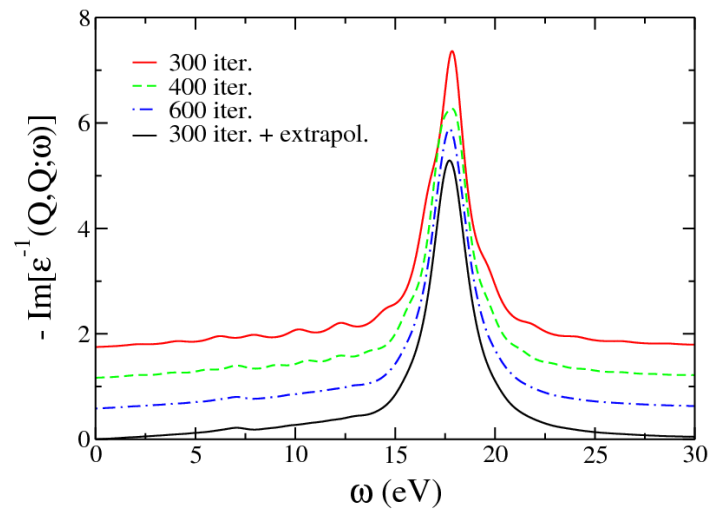

(a)

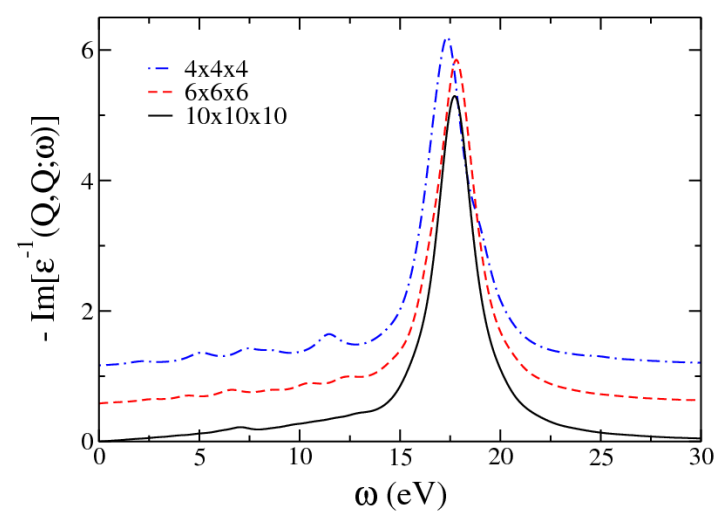

(b)

FIG. 3: Bulk Al: Loss function calculated for $Q=0.513$ a.u. along [100]. (a) Convergence with respect to the number of Lanczos iterations, using a $10 \times 10 \times 10 \mathrm{MP}$ k point mesh, and effect of the extrapolation technique. (b) Convergence with respect to the size of the $\mathbf{k}$ point mesh, using 600 Lanczos iterations. Both figures have been obtained with a Lorentzian broadening $\eta=0.056 \mathrm{Ry}$. Curves have been shifted vertically for clarity.

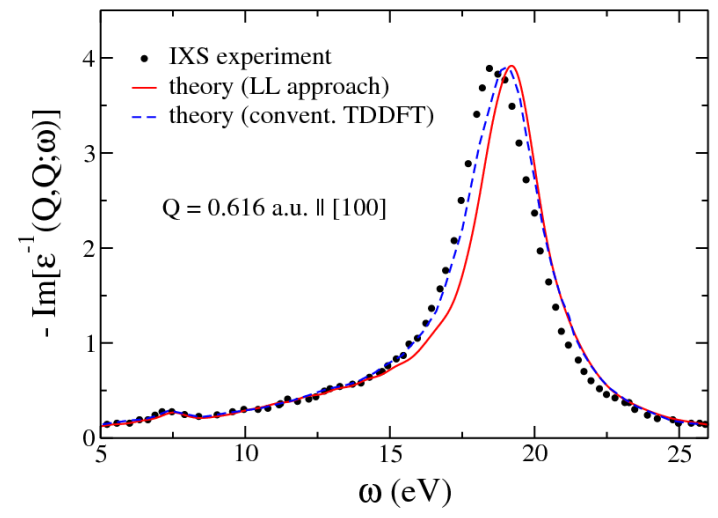

(a)

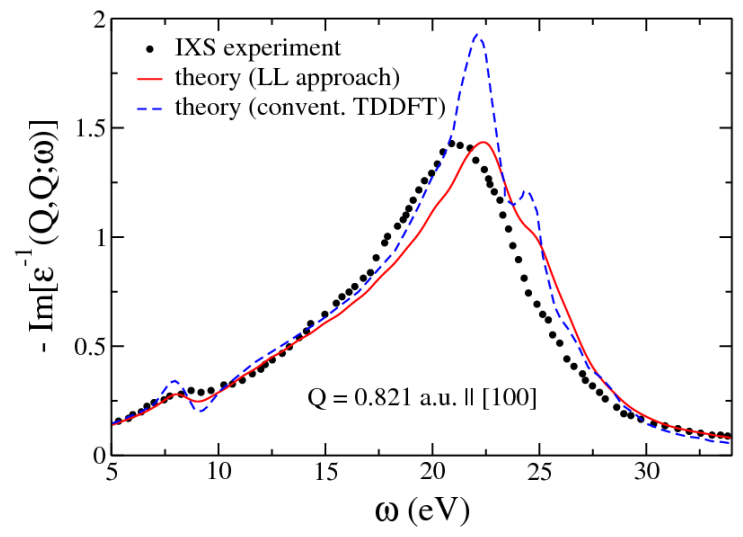

(b)

FIG. 4: Bulk Al: Comparison of the loss function calculated for two different values of the transferred momentum, $\mathbf{Q}$, using the Liouville-Lanczos (LL) approach, with experiment $\frac{47}{4}$ and with previous theory $\frac{47}{4}$ (a) $Q=0.616$ a.u. along [100], (b) $Q=0.821$ a.u. along [100]. LL data have been obtained using 400 Lanczos iterations plus extrapolations. A $10 \times 10 \times 10$ $(14 \times 14 \times 14)$ MP k point mesh and a Lorentzian broadening $\eta$ of 0.051 (0.068) Ry have been used for the two cases, respectively.

work. At small transferred momentum (panel (a)) theoretical predictions agree remarkably well with each other (the slight discrepancies being attributable to the usual small differences between the technical details of the calculations) and with experiment. Both theoretical spectra display a small blueshift $(\sim 0.5 \mathrm{eV})$ of the plasmon peak with respect to experiments. At larger transferred momentum (panel (b)) the theoretical spectra display a feature at $\sim 24 \mathrm{eV}$, which is not observed experimentally. We attribute the remaining discrepancies to the lifetime effects $\frac{47}{17}$ which have been treated in our calculations by a constant Lorentzian broadening parameter $(\eta=0.068 \mathrm{Ry}$, requiring a $14 \times 14 \times 14 \mathrm{MP}$ mesh $)$.

\section{CONCLUDING REMARKS}

We believe that the Liouville-Lanczos approach introduced in this paper will open new perspectives in the calculation of loss spectra in extended systems. Its main features are the adoption of a representation for the chargedensity response borrowed from density-functional perturbation theory, and of a Lanczos recursion scheme for computing selected elements of the inverse of (very) large matrices. The combination of these two elements permits to compute the loss spectrum of a given system, for a given transferred momentum, and for an entire wide frequency range, with a numerical workload of the same order as needed for a standard ground-state calculation for a same system (the pre-factor being only a 
few times larger). In principle, the convergence of the computed loss spectra with respect to the length of the Lanczos chains depends on the spectral range: the lower the frequency, the faster the convergence, as it was already observed for optical spectra in finite systems. $\underline{\square 0 \mid 51}$ In practice, however, adoption of the extrapolation techniques explained in Sec. II B3 substantially alleviates this dependence. Also, the spectral range accessible to EEL/IXS spectroscopies is limited by the so-called $f$-sum rule!

$$
\int_{0}^{\infty} \operatorname{Im}\left[\epsilon^{-1}(\mathbf{Q}, \mathbf{Q} ; \omega)\right] \omega d \omega=-\frac{\pi}{2} \omega_{p}^{2}
$$

where $\omega_{p}=4 \pi e^{2} n_{e} / m$ is the plasma frequency, $n_{e}$ being the average electron density, i.e. the number of electrons (valence electrons, in a pseudopotential calculation) per unit volume ${ }^{80}$ Of course, the spectral range that needs to be sampled by Lanczos recursion is correspondingly limited.

The Liouville-Lanczos approach introduced in this paper also lends itself to an easy generalization to those methods (such as hybrid functionals or the static BetheSalpeter equation - BSE) that require the full densitymatrix (rather than just charge-density) response, which is in fact as easily accessible to the batch representation utilized here ${ }^{81}$ Further generalization to frequencydependent $\mathrm{XC}$ kernels (or to the BSE with dynamical screening) may simply require computing the loss function at shifted frequencies $\left(\omega^{\prime}=\omega+\Sigma(\omega)\right)$, as proposed e.g. in Ref. 46, or further methodological developments. Further work is required to clarify this issue.

All in all we believe that the advances presented in this paper will allow for the simulation of complex, possibly nano-structured, surfaces, as well as of systems where valence and shallow-core loss spectra overlap. Examples of the former include low Miller index surfaces or plasmonic materials, while bulk bismuth is an example of the latter. Work is in progress on both lines.

\section{ACKNOWLEDGMENTS}

We thank S. de Gironcoli, A. Dal Corso, and L. Reining for valuable discussions. Support from the ANR (Project PNANO ACCATTONE) and from DGA are gratefully acknowledged. Computer time was granted by GENCI (Project No. 2210). The work of I.T. and N.V. has been performed under the auspices of the Laboratoire d'excellence en nanosciences et nanotechnologies Labex Nanosaclay. N.V. thanks Marco Saitta for discussions about TDDFPT at an early stage of the project. S.B. gratefully acknowledges hospitality at the Laboratoire des Solides Irradiés of the École Polytechnique, where this paper was written.
1 D. Pines, Can. J. Phys. 34, 1379 (1956).

2 P. Nozières and D. Pines, Phys. Rev. 113, 1254 (1959).

3 D. Pines, Elementary excitations in solids (New York, 1964).

${ }^{4}$ R. F. Egerton, Electron Energy-Loss Spectroscopy in the Electron Microscope (Plenum, New York and London, 1996), 2nd ed.

5 W. Schülke, Electron Dynamics by Inelastic X-Ray Scattering (Oxford University Press, Oxford, 2007).

${ }^{6}$ H. A. Atwater and A. Polman, Nat. Mater. 9, 205 (2011).

7 T. Bartal, M. Foord, C. Bellei, M. Key, K. Flippo, S. Gaillard, D. Offermann, P. Patel, L. Jarrott, D. Higginson, et al., Nature Physics 8, 139 (2011).

8 J. N. Anker, W. P. Hall, O. Lyandres, N. C. Shah, J. Zhao, and R. P. V. Duyne, Nat. Mater. 7, 442 (2008).

9 V. M. Silkin, J. M. Pitarke, E. V. Chulkov, and P. M. Echenique, Phys. Rev. B 72, 115435 (2005).

10 B. Diaconescu, K. Pohl, L. Vattuone, L. Savio, P. Hofmann, V. Silkin, J. Pitarke, E. Chulkov, P. Echenique, D. Farias, et al., Nature 448, 57 (2007).

11 K. Pohl, B. Diaconescu, G. Vercelli, L. Vattuone, V. Silkin, E. Chulkov, P. Echenique, and M. Rocca, European Physics Letters 90, 57006 (2010).

12 L. Vattuone, G. Vercelli, M. Smerieri, L. Savio, and M. Rocca, Plasmonics 7, 323 (2012).

13 J. Yan, K. Jacobsen, and K. Thygesen, Phys. Rev. B 86, 241404(R) (2012).

14 L. Vattuone, M. Smerieri, T. Langer, C. Tegenkamp,
H. Pfnür, V. M. Silkin, E. V. Chulkov, P. M. Echenique, and M. Rocca, Phys. Rev. Lett. 110, 127405 (2013).

15 V. M. Silkin, A. García-Lekue, J. M. Pitarke, E. V. Chulkov, E. Zaremba, and P. M. Echenique, Europhys. Lett. 66, 260 (2004).

16 J. Pitarke, V. U. Nazarov, V. Silkin, E. Chulkov, E. Zaremba, and P. Echenique, Phys. Rev. B 70, 205403 (2004).

17 J. Pitarke, V. Silkin, E. Chulkov, and P. Echenique, Rep. Prog. Phys. 70, 1 (2007).

18 R. Esteban, A. Borisov, P. Nordlander, and J. Aizpurua, Nature Communications 3, 825 (2012).

19 K. Sturm, Phys. Rev. Lett. 40, 1599 (1978).

20 M. Cohen and T. K. Bergstresser, Phys. Rev. 141, 789 (1966).

21 S. G. Louie, J. Chelikowsky, and M. L. Cohen, Phys. Rev. Lett. 34, 155 (1975).

22 E. Runge and E. Gross, Phys. Rev. Lett. 52, 997 (1984).

23 E. K. U. Gross, J. F. Dobson, and M. Petersilka, Density Functional Theory of Time-Dependent Phenomena, Topics in Current Chemistry (Springer-Verlag, Berlin, 1996).

${ }^{24}$ W. Caliebe, J. Soininen, E. Shirley, C.-C. Kao, and K. Hämäläinen, Phys. Rev. Lett. 84, 3907 (2000).

25 V. Olevano and L. Reining, Phys. Rev. Lett. 86, 5962 (2001).

26 Y. Takada and H. Yasuhara, Phys. Rev. Lett. 89, 216402 (2002).

27 B. Arnaud, S. Lebègue, and M. Alouani, Phys. Rev. B 71, 
035308 (2005).

28 R. Daling, W. van Haeringen, and B. Farid, Phys. Rev. B 45, 8970 (1992).

29 G. Engel and B. Farid, Phys. Rev. B 46, 15812 (1992).

${ }^{30}$ K. Sturm, W. Schülke, and J. Schmitz, Phys. Rev. Lett. 68, 228 (1992).

31 A. A. Quong and A. Eguiluz, Phys. Rev. Lett. 70, 3955 (1993).

32 A. Fleszar, A. Quong, and A. Eguiluz, Phys. Rev. Lett. 74, 590 (1995).

33 M. Ehrnsperger and H. Bross, J. Phys.: Condens. Matter 9, 1225 (1997).

34 S. Waidmann, M. Knupfer, B. Arnold, J. Fink, A. Fleszar, and W. Hanke, Phys. Rev. B 61, 10149 (2000).

35 N. Vast, L. Reining, V. Olevano, P. Schattschneider, and B. Jouffrey, Phys. Rev. Lett. 88, 037601 (2002).

36 A. Marinopoulos, L. Reining, V. Olevano, A. Rubio, T. Pichler, X. Liu, M. Knupfer, and J. Fink, Phys. Rev. Lett. 89, 076402 (2002).

37 A. Marinopoulos, L. Reining, A. Rubio, and N. Vast, Phys. Rev. Lett. 91, 046402 (2003).

${ }^{38}$ W.-D. Schöne, D. S. Su, and W. Ekardt, Phys. Rev. B 68, 115102 (2003).

39 L. Dash, N. Vast, P. Baranek, M.-C. Cheynet, and L. Reining, Physical Review B 70, 245116 (2004).

40 I. G. Gurtubay, W. Ku, J. M. Pitarke, A. G. Eguiluz, B. C. Larson, J. Tischler, and P. Zschack, Phys. Rev. B 70, 201201(R) (2004).

${ }^{41}$ I. G. Gurtubay, J. M. Pitarke, W. Ku, A. G. Eguiluz, B. C. Larson, J. Tischler, P. Zschack, and K. D. Finkelstein, Phys. Rev. B 72, 125117 (2005).

${ }^{42}$ H.-C. Weissker, J. Serrano, S. Huotari, F. Bruneval, F. Sottile, G. Monaco, M. Krisch, V. Olevano, and L. Reining, Phys. Rev. Lett. 97, 237602 (2006).

${ }^{43}$ C. Kramberger, R. Hambach, C. Giorgetti, M. Rümmeli, M. Knupfer, J. Fink, B. büchner, L. Reining, E. Einarsson, S. Maruyama, et al., Phys. Rev. Lett. 100, 196803 (2008).

44 A. Alkauskas, S. Schneider, S. Sagmeister, C. AmbroschDraxl, and C. Hébert, Ultramicroscopy 110, 1081 (2010).

45 S. Huotari, C. Sternemann, M. Troparevsky, A. Eguiluz, M. Volmer, H. Sternemann, H. Müller, G. Monaco, and W. Schülke, Phys. Rev. B 80, 155107 (2009).

46 H.-C. Weissker, J. Serrano, S. Huotari, E. Luppi, M. Cazzaniga, F. Bruneval, F. Sottile, G. Monaco, V. Olevano, and L. Reining, Phys. Rev. B 81, 085104 (2010).

47 M. Cazzaniga, H.-C. Weissker, S. Huotari, T. Pylkkänen, P. Salvestrini, G. Monaco, G. Onida, and L. Reining, Phys. Rev. B 84, 075109 (2011).

48 J. Yan, K. S. Thygesen, and K. W. Jacobsen, Phys. Rev. Lett. 106, 146803 (2011).

49 G. Onida, L. Reining, and A. Rubio, Rev. Mod. Phys. 74, 601 (2002).

50 B. Walker, A. M. Saitta, R. Gebauer, and S. Baroni, Phys. Rev. Lett. 96, 113001 (2006).

51 D. Rocca, R. Gebauer, Y. Saas, and S. Baroni, J. Chem. Phys. 128, 154105 (2008).

52 O. B. Malcioglu, R. Gebauer, D. Rocca, and S. Baroni, Comput. Phys. Commun. 182, 1744 (2011).

53 S. Baroni and R. Gebauer, Fundamentals of TimeDependent Density Functional Theory (Springer, Berlin, 2012).

54 J. A. Soininen, A. L. Ankudinov, and J. J. Rehr, Phys. Rev. B 72, 045136 (2005).

55 Y. Joly, Phys. Rev. B 63, 125120 (2001).
${ }^{56}$ D. Cabaret, N. Emery, C. Bellin, C. Herold, P. Lagrange, F. Wilhelm, A. Rogalev, and G. Loupias, Phys. Rev. B 87, 075108 (2013).

57 S. Baroni, S. de Gironcoli, A. Dal Corso, and P. Giannozzi, Rev. Mod. Phys. 73, 515 (2001).

${ }^{58}$ L. V. Hove, Phys. Rev. 95, 249 (1954).

59 H.-K. Mao, C. Kao, and R. Hemley, J. Phys.: Condens. Matter 13, 7847 (2001).

60 I. Loa, K. Syassen, G. Monaco, G. Vankò, M. Krish, and M. Hanfland, Phys. Rev. Lett. 107, 086402 (2011).

61 D. Pines and P. Nozières, The Theory of Quantum Liquids, vol. 1 (Benjamin, New York, 1966).

62 R. Martin, ed., Electronic Structure: Basic Theory and Practical Methods (Cambridge University Press, Cambridge, 2004).

63 R. Car, E. Tosatti, S. Baroni, and S. Leelaprute, Phys. Rev. B 24, 985 (1981).

64 E. K. U. Gross and W. Kohn, Phys. Rev. Lett. 55, 2850 (1985).

65 According to Eq. (10), the response density matrix is uniquely identified by the two sets of $N_{v}$ response orbitals $\left\{\varphi_{v}^{\prime}(\mathbf{r}, \pm \omega)\right\}$, each one of which is a linear combination of $N_{c}$ virtual orbitals.

66 D. W. Bullet, R. Haydock, V. Heine, and M. Kelly, Solid State Physics, vol. 35 (Academic, New York, 1980).

67 Y. Saad, Iterative Methods for Sparse Linear Systems (SIAM, Philadelphia, 2003), 2nd ed.

68 A. L. Ankudinov, C. E. Bouldin, J. J. Rehr, J. Sims, and H. Hung, Phys. Rev. B 65, 104107 (2002).

69 M. Grüning, A. Marini, and X. Gonze, Comp. Mater. Sci. 50, 2148 (2011).

70 I. Timrov, Ph.D. thesis, École Polytechnique, France (2013), URL http://pastel.archives-ouvertes.fr/ pastel-00823758

${ }^{\prime}$ S. de Gironcoli, Phys. Rev. B 51, 6773 (1995).

72 P. Giannozzi, S. Baroni, N. Bonini, M. Calandra, R. Car, C. Cavazzoni, D. Ceresoli, G. Chiarotti, M. Cococcioni, I. Dabo, et al., J. Phys.: Condens. Matter 21, 395502 (2009).

73 J. Perdew and A. Zunger, Phys. Rev. B 23, 5048 (1981).

74 http://www.quantum-espresso.org/pseudopotentials Si.pz-vbc.UPF and Al.pz-vbc.UPF.

75 M. Methfessel and A. Paxton, Phys. Rev. B 40, 3616 (1989).

76 M. Neuberger, Handbook of Electronic Materials (Plenum, New York, 1971).

77 R. W. Wyckoff, Crystal Structures, vol. 1 (John Wiley and Sons Ltd, New York, 1963), 2nd ed.

78 G. D. Mahan, Many-particles physics (Plenum Press, New York, 1975), 2nd ed.

79 The dynamic structure factor $S(\mathbf{Q}, \omega)$ has been transformed to $-\operatorname{Im}\left[\epsilon^{-1}(\mathbf{Q}, \mathbf{Q} ; \omega)\right]$ using Eq. (3) of Ref. 46, with the electron density being equal to 0.03 (a.u. $)^{-3}$.

80 A remarkable feature of the Liouville-Lanczos approach is that the $f$-sum rule is satisfied exactly when truncating the Lanczos recursion to any number of iterations. ${ }^{[53}$ The validity of this sum rule relies on the locality of the external potential. When non-local pseudopotentials are used, which is usually the case with plane-wave basis sets, violations of the $f$-sum rule occur, $\frac{52]}{}$ which we found to be sensitive to the accuracy of Brillouin zone sampling. In the present case we found these violations to be smaller than $7 \%$. 
${ }^{81}$ D. Rocca, Y. Ping, R. Gebauer, and G. Galli, Phys. Rev. B 85, 045116 (2012). 